\title{
Quantification and influence of skin chromophores for remote detection of anemic conditions
}

Kallepalli, Akhil, James, David

Akhil Kallepalli, David B. James, "Quantification and influence of skin chromophores for remote detection of anemic conditions," Proc. SPIE 11238, Optical Interactions with Tissue and Cells XXXI, 112381B (20 February 2020); doi: $10.1117 / 12.2545784$

SPIE. Event: SPIE BiOS, 2020, San Francisco, California, United States 


\title{
Quantification and influence of skin chromophores for remote detection of anemic conditions
}

\author{
Akhil Kallepalli ${ }^{*}$ and David B. James ${ }^{\mathrm{a}}$ \\ ${ }^{a}$ Centre for Electronic Warfare, Information and Cyber, Cranfield University, Defence \\ Academy of the UK, Shrivenham SN6 8LA, United Kingdom
}

\begin{abstract}
Current standards for diagnosing and monitoring anemia are relatively invasive. The superficial symptoms of this condition are due to an underlying deficiency of red blood cells (RBC) or erythrocytes, and hemoglobin in the blood. This results in an inadequate supply of oxygen to the body's tissues. For point-of-care diagnostic systems, remote determination of blood conditions will depend on an understanding of the interaction of light with hemoglobin. However, the skin acts as the first barrier for this detection. In this study, we pursue the possibility of detecting anemic conditions from the perfused blood in the dermis using optical models and Monte Carlo (MC) methods.

The skin is composed of two primary layers, the epidermis and the dermis. The avascular epidermis absorbs light due to its primary chromophore, melanin. Subsequently, the absorption in the dermis layer is quantified by hematocrit and hemoglobin concentrations. Two-layer models of the human skin are set up and optical properties are assigned to these models. The optical variability across these models are defined by six melanin (epidermis) and two erythrocytes (dermis) concentrations. The twelve combinations of optical properties are assessed at six wavelengths of interest in the Virtual Tissue Simulator (VTS) environment.

The chosen wavelengths range across the visible and near-infrared spectrum, which is a known and important diagnostic window for biological tissues. In this study, we explore the variability of light interactions for healthy and anemic blood conditions quantified in the dermis while accounting for variable melanin concentrations in the epidermis.
\end{abstract}

Keywords: Monte Carlo, chromophores, blood perfusion, diagnostics, melanin, hemoglobin, blood perfusion, attenuation coefficients

\section{INTRODUCTION}

Anemia is a condition resulting from a lower than normal amount of erythrocytes/red blood cells (RBCs) and/or hemoglobin in the blood. While blood tests are the current standard for accurately measuring the changes in the hemoglobin concentration, they are invasive and can be uncomfortable. Additionally, should anemic conditions present themselves post-trauma or during surgery, monitoring by repeatedly extracting blood is not practical. Symptoms are usually related to the level of fluctuating hemoglobin, the onset rapidity and overall duration. Such conditions, if undiagnosed, could lead to long-term health problems. Using optical techniques to investigate the blood conditions allow the advantage of continuous monitoring, coupled with the benefits of a non-invasive method for diagnosis. In this research article, we present an analysis of photon transport through the skin layers, motivated by the need to assess blood conditions for point-of-care diagnostics.

The current, most commonly used diagnostics tool for blood monitoring is the pulse oximeter. It monitors the oxygen saturation and heart rate, exploiting the variable absorption of red and near-infrared wavelengths with blood. As the body modulates the RBC volume to ensure adequate blood supply to tissues, tissue oxygen supply remains relatively constant and the underlying variations of $\mathrm{RBC}$ volume remain undetected. This can be dangerous because in cases such as internal bleeding from tumors or stomach ulcers, ${ }^{1}$ the resulting loss of

*AK (Corresponding author)

Email: a.kallepalli@cranfield.ac.uk, kallepalliakhil@icloud.com

Optical Interactions with Tissue and Cells XXXI, edited by Bennett L. Ibey, Norbert Linz, Proc. of SPIE

Vol. 11238, 112381B · @ 2020 SPIE · CCC code: 1605-7422/20/\$21 · doi: 10.1117/12.2545784 
iron in the blood cannot be detected by oximeters. Herein, lies the need for a blood monitoring system with the capability of detecting RBC volume and/or hemoglobin variation.

This study presents a simulation-based analysis of skin models for detection of anemia. Using Monte Carlo (MC) methods, photon transport through two-layer skin models (epidermis, dermis) is simulated at six wavelengths in the visible and near-infrared region of the electromagnetic spectrum. The avascular epidermis is assigned optical properties to represent the six skin types according to the Fitzpatrick scale. ${ }^{2}$ The dermis layer consists of perfused blood and the optical properties represent the healthy and anemic conditions through quantified hematocrit and volume fraction of RBCs in the blood. The combination of six melanin concentrations in the epidermis and two dermal perfusion conditions at six wavelengths result in 72 scenarios for assessment.

\section{BACKGROUND}

Hemoglobin is a key protein present primarily within RBCs and is responsible for the transport of oxygen to tissues. It is made up of two pairs of dissimilar chains, $\alpha$ and $\beta$, which are specific amino acid sequences containing a 'heme' group. The combination of both chains and a consequent 'heme-heme' interaction facilitates oxygen uptake (oxygenation of hemoglobin) and delivery (deoxygenation of hemoglobin). ${ }^{1}$ In the case of an abnormal shift in the oxygen dissociation curve, the body adjusts the hemoglobin levels through erythropoiesis (production of RBCs in the bone marrow) and ensures adequate oxygen supply to the tissues. This modulation averts tissue hypoxia (lack of oxygen).

The symptoms of abnormal hemoglobin levels are non-specific; fatigue, pale appearance, palpitations and shortness of breath are a result of either decrease in the production or increase in loss/destruction of RBCs, resulting in an overall reduction of hemoglobin in the blood. Due to the nature of these symptoms, most anemic conditions go undiagnosed until the symptoms are more severe. The current test for anemia is based on measuring the mean corpuscular volume (MCV) which quantifies the average volume of RBCs in the blood. Blood tests that measure the complete blood count (CBC) facilitate not only a diagnosis, but also decipher the cause of the condition. Blood health within the context of anemia (and in this study) is defined with three quantities: hematocrit $(H t)$, the ratio of RBCs to the total cell volume $\left(F_{R B C}\right)$ and the amount of hemoglobin $(\mathrm{Hb})$ in individual RBCs $\left(F_{H b}\right)$. Hematocrit $(H t)$ is the ratio of the volume of RBCs compared to the total blood volume (blood cells and plasma). For sufficient oxygen supply to the tissues, the hemoglobin levels in the blood must be approximately $16 \mathrm{~g} / \mathrm{dl}$ for male or $14 \mathrm{~g} / \mathrm{dl}$ for female individuals. ${ }^{1}$ Normal hematocrit levels are, on average, $47 \%$ for male and $42 \%$ for female individuals and result in healthy tissue oxygen delivery. Blood is predominantly made up of cells and plasma, with the RBCs being almost $99 \%$ of the cells in the blood. Majority of the hemoglobin in the blood is found enclosed in RBCs, with an average volume fraction of $25 \%$ in each $\mathrm{RBC}^{*}$.

In order to diagnose/monitor anemic conditions in a non-invasive method using optical methods, the photon transport through tissues in the optical diagnostic window of visible and near-infrared wavelengths ${ }^{3}$ must be understood. Each tissue behaves characteristically when interacting with photons of specific wavelengths. ${ }^{4,5}$ This behavior is quantified by optical properties, which are:

- Absorption coefficient, $\mu_{a}\left(\mathrm{~mm}^{-1}\right)$

- Scattering coefficient, $\mu_{s}\left(\mathrm{~mm}^{-1}\right)^{\dagger}$

- Refractive index, $\eta(-)$

- Anisotropy, $g(-)$

- Reduced scattering coefficient, $\mu_{s}^{\prime}=\mu_{s}(1-g)\left(\mathrm{mm}^{-1}\right)$

A fraction of the photon's energy is lost due to absorption in the biological medium. This absorption increases exponentially as a function of increasing pathlength of the photon $(L)$, concentration $(C)$ and extinction coefficient $(\varepsilon)$ for the chromophore (transmission, $T=10^{\varepsilon C L}$ ). With known concentrations, the absorption coefficient is calculated as a sum of all contributing chromophores in the tissue $\left(\mu_{a}=\log _{1} 0 \Sigma C_{i} \varepsilon_{i}\right)$. In the

${ }^{*}$ Hemoglobin within the blood is variable in terms of size, concentration, cells flowing through a body site, and are also found in the bloodstream outside RBCs. However, the percentage of hemoglobin outside RBCs is relatively low.

${ }^{\dagger}$ The sum total of absorption $\left(\mu_{a}\right)$ and scattering $\left(\mu_{s}\right)$ coefficients is known as the attenuation coefficient $\left(\mu_{t}\right)$. 
absence of molecular compositions of the tissue, absorption coefficient can be defined in terms of volume fractions of the tissue components, $v$, and their corresponding absorption coefficients $\left(\mu_{a}=\Sigma f_{v} \mu_{a . v}\right){ }^{4}$ Transmission measurements using integrating spheres and inverse Monte Carlo (MC) methods are commonly used to measure the absorption coefficient.

Scattering coefficient, as itself, is difficult to measure. It requires the sample to be prepared to the scale of one mean free path, which is typically $100 \mu \mathrm{m}$ or less. At this scale, the heterogeneity of the tissue, desiccation of the sample and the solid angle of collection of the detector cause underestimation of the scattering coefficient. Similarly, the measurement of $g$ is also difficult. Although possible through goniometry and indirect methods using diffuse and collimated light for estimating $\mu_{s}^{\prime}$ and $\mu_{s}$, respectively, the measures are not robust. ${ }^{4}$ Thus, most studies measure the reduced scattering coefficient through comparatively simpler methods, such as using integrating spheres. The anisotropy factor is a contributing factor to the Henyey-Greenstein phase function. ${ }^{6}$ The values $-1 \leq g \leq 1$ indicates the nature of scattering, with $-1,0$ and 1 indicating backscatter, isotropic scattering and forward scatter, respectively.

One of the initial skin optics studies assessed the transmission and reflectance from the epidermis and dermis layers across the visible and infrared spectrum. ${ }^{7}$ The study also showed the differences in attenuation for different skin types at the extremes of the Fitzpatrick scale. The contributions of blood in the dermis layer are discussed. However, the blood and water absorption peaks were missing and absorption was overestimated while the scattering was underestimated. ${ }^{8}$ Similarly, there is a continuing debate about the variability and applicability of optical properties. In the many techniques of sample preparation and optical measurements, there is a 100fold variability in the reported optical property values. ${ }^{8}$ After screening the optical properties, a subset of the values are analyzed using MC methods and compared to standards from the National Institute of Standards and Technology (NIST). This method of screening the optical properties' values was done in our current study and is discussed in Section 3.

Theoretical and analytical methods are used when solutions of photon-tissue interactions are required. Of these, the primary model is the radiative transfer equation (RTE). ${ }^{9}$ Although it applies to only numerical solutions and in simple cases, the RTE is the best theoretical solution for combining losses due to attenuation and gains due to reflection and scattering towards the source. Due to its innate disadvantages, the RTE is approximated to the diffusion or $\mathrm{P}_{1}$ approximation using spherical harmonics. However, the diffusion approximation considers propagation to be isotropic and therefore, in cases where directionality is critical, the solution of the diffusion approximation is not suitable. ${ }^{5}$ Therefore, the stochastic MC method has emerged as the current "gold standard" for modeling photon propagation through biological tissue. Although computationally demanding and timeconsuming, this method presents advantages of simultaneous estimation of multiple physical quantities and holding true to directionality. ${ }^{10}$

One of the early implementations of MC methods was presented by Wang et al. (1995), ${ }^{11}$ known as Monte Carlo modeling of light transport in multi-layered tissues (MCML). Simulation of photon propagation and the role of optical attenuation coefficients are discussed. The results are compared to Prahl et al. (1989), ${ }^{12}$ who previously presented the implementation of MC programs, inclusion of variance reduction schemes and consideration of flat and Gaussian sources. MC methods have since been utilized for many biological applications. Photon interactions with blood vessels in the skin were studied ${ }^{13}$ and a key observation of the penetration of red wavelengths of light through to the center of the blood vessels was made. Other studies modeled the skin layers to include the constituent layers, perfused blood and hypodermic fat (which is present in body regions with thicker skin). ${ }^{14}$ This study forms the basis and reasoning behind the subsequent study, Meglinski and Matcher (2002), ${ }^{15}$ which is used for dermal absorption coefficient calculation in this current study. Examples of implementation of MC methods for skin lesion models, ${ }^{16}$ estimating tissue optical properties for synthetic phantoms which are used for breast cancer diagnosis, ${ }^{17,18}$ extending and assessing more detailed skin models such as nine-layer skin models and comparison with in vivo skin tissue ${ }^{19}$ and being applied to optical coherence tomography retinal oximetry ${ }^{20}$ have validated its applicability to biological problems. A review of the various MC methods has been presented by Zhu and Liu $(2013)^{21}$ and is recommended for further reading. 


\section{METHODOLOGY}

The methodology of this study includes attributing different melanin concentrations in the epidermis layer depicting the skin types according to the Fitzpatrick scale and blood conditions for the dermal blood distribution to the tissue models. Following which, MC methods are used in order to assess the reflectance, fluence, transmittance and source-detector scenarios for detection of reflected photons from the two-layer skin models. The sources are simulated to emit photons at six wavelengths, namely $515 \mathrm{~nm}, 565 \mathrm{~nm}, 615 \mathrm{~nm}, 665 \mathrm{~nm}, 750 \mathrm{~nm}$ and $950 \mathrm{~nm}$. A subset of the optical property values reported and models for the property calculation are adopted in this study after screening the literature on a case-by-case basis. The choice is a key step in assessing the optical models as the original study's characteristics could influence the assessment.

In biological tissues, the primary causes of attenuation are the chromophores and constituent structures. In the case of skin, absorption in the layers is due to the individual layers' primary chromophores, melanin (epidermis) and hemoglobin (dermis). Scattering of photons in the layers is due to the keratin and collagen fibers, respectively. Therefore, the optical property values of the epidermis and dermis layers for this study must account for:

- absorption due to melanin and its degree, which is dependent on the melanin in the avascular epidermis layer.

- the influence of blood and the degree of absorption in the dermis layers.

- scattering in the epidermis and dermis layers due to collagen fibers and other constituent structures.

\subsection{Absorption Coefficients of the Skin Layers}

The absorption in the epidermis layer is due to melanin and its concentration ${ }^{4,22}$ distributed in the terminally differentiated layers of the epidermis. The absorption coefficient of the epidermis layer $\left(\mu_{\text {a.epidermis }} ;\right.$ Eq. 3$)$ is defined with respect to skin without chromophores $\left(\mu_{\text {a.skin }} ;\right.$ Eq. 1$)$ and melanin distribution $\left(\mu_{a . m e l a n i n}\right.$; Eq. 2). The absorption coefficient for baseline skin was calculated in vitro from neonatal skin samples using an integrating sphere by Saidi (1992). ${ }^{4,23}$ The dependence of absorption on the concentration of melanin is accounted for as a volume fraction factor $\left(f_{\text {melanin }}\right)$ in equation 3.

$$
\begin{gathered}
\mu_{\text {a.skin }}=7.84 \times 10^{7} \times \lambda^{-3.255}[\lambda: n m] \\
\mu_{\text {a.melanin }}=6.6 \times 10^{10} \times \lambda^{-3.3}[\lambda: n m] \\
\mu_{\text {a.epidermis }}=\left(f_{\text {melanin }}\right)\left(\mu_{\text {a.melanin }}\right)+\left(1-f_{\text {melanin }}\right)\left(\mu_{\text {a.skin }}\right)
\end{gathered}
$$

Table 1. Anemic manifestation in the perfused blood in the dermis layer is simulated by varying the hematocrit $(H t)$ and the RBCs vol. frac. $\left(F_{R B C}\right)$. Although relatively extreme, a clear manifestation of anemia assists in identifying the appropriate markers for remote detection. The volume fraction of melanin distribution in the epidermis considered in this study is also presented.

\begin{tabular}{|c|c|c|c|c|c|}
\hline \multicolumn{5}{|c|}{$\begin{array}{c}\text { Epidermal Melanin Vol. Frac. } \\
\text { in Different Skin Types }\end{array}$} \\
\hline I & II & III & IV & V & VI \\
\hline 0.029 & 0.045 & 0.126 & 0.142 & 0.263 & 0.346 \\
\hline \multicolumn{5}{|c|}{ Dermal Perfusion } \\
Vol. Frac. \\
\hline \multicolumn{5}{|c|}{ Healthy } & \multicolumn{3}{c|}{ Anemic } \\
\hline Ht & 0.45 & Ht & 0.34 \\
\hline$F_{R B C}$ & 0.99 & $F_{R B C}$ & 0.75 \\
\hline$F_{H b}$ & 0.25 & $F_{H b}$ & 0.25 \\
\hline
\end{tabular}


The absorption of photons in the epidermis in this study is calculated using the baseline skin properties, optical properties of melanin and the volume fraction of melanin in the layer (Eq. 3). The volume fraction of melanin $\left(f_{\text {melanin }}\right)$ in the epidermis layer is based on the Fitzpatrick scale and the values used to characterize the skin types are given in Table 1. The wavelengths of interest in this study $(515 \mathrm{~nm}, 565 \mathrm{~nm}, 615 \mathrm{~nm}, 665$ $\mathrm{nm}, 750 \mathrm{~nm}$ and $950 \mathrm{~nm}$ ) are combined with the volume fraction of melanin in the epidermal layer $\left(f_{\text {melanin }}\right)$ in equations 1-3. The calculated absorption coefficient values for the epidermis are given in Figure 1.

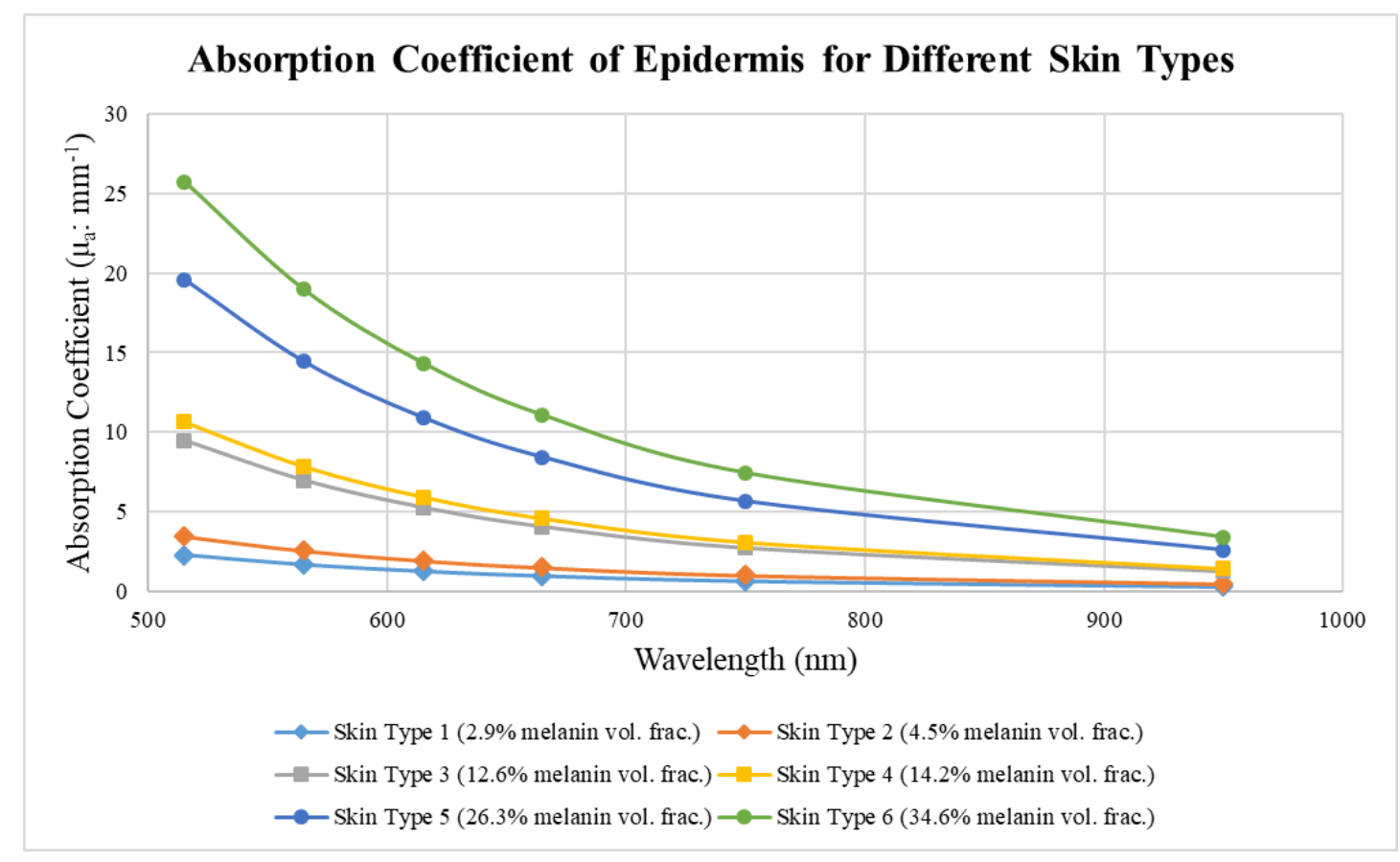

Figure 1. The absorption coefficient of the epidermis $\left(\mu_{\text {a.epidermis }}\right)$ is calculated for different volume fractions of melanin at the six wavelengths of interest. The volume fractions represent the different skin types and are used to calculate the fraction of photon absorption in the layer.

The absorption properties for the dermis layer are calculated by accounting for the constituents of the layer, namely, baseline skin, blood and water. The contribution of oxygenated and deoxygenated hemoglobin at different wavelengths must also be considered ${ }^{15}$ (Eq. 5).

$$
\begin{gathered}
\gamma=H t \times F_{R B C} \times F_{H b} \\
\mu_{\text {a.dermis }}=(1-S) \gamma C_{\text {blood }} \mu_{a . H b}(\lambda)+S \gamma C_{\text {blood }} \mu_{a . H b O 2}(\lambda) \\
+\left(1-\gamma C_{\text {blood }}\right) C_{H 2 O} \mu_{a . H 2 O}(\lambda)+\left(1-\gamma C_{b l o o d}\right)\left(1-C_{H 2 O}\right) \mu_{a . s k i n}
\end{gathered}
$$

The absorption in the dermis layer is quantified using several parameters ${ }^{15}$ (as seen in Eq. 5). The terms in the model, with constant values considered for this study where mentioned, are:

- oxygen saturation, $S=0.6$;

- the common factor, $\gamma$, which accounts for hematocrit $(H t)$, cell volume fraction of RBCs in the blood $\left(F_{R B C}\right)$ and volume fraction of hemoglobin in individual RBCs $\left(F_{H b}\right)$;

- concentration of blood, $C_{\text {blood }}=0.04$;

- concentration of water, $C_{H 2 O}=0.6$ 
Discussing the individual terms of the relationship (Eq. 5) as a function of wavelength $(\lambda)$ :

- the first term accounts for the absorption by deoxygenated blood $\left(\mu_{a . H b}(\lambda)\right)$;

- the second for absorption by oxygenated blood $\left(\mu_{a . \mathrm{HbO} 2}(\lambda)\right)$;

- the third term accounts for absorption due to water $\left(\mu_{a . H 2 O}(\lambda)\right)$ in the layer;

- the final term accounts for absorption due to the baseline skin $\left(\mu_{a . s k i n} ;\right.$ Eq. 1$)$.

The individual absorption coefficients of deoxygenated blood, oxygenated blood and water at the specific wavelengths used in this study are adopted from Jacques $(2013)^{4}$ and the blood condition is quantified in equation 4. Under healthy conditions, the absorption properties of the dermis are calculated and shown in Figure 2 (triangles). In order to simulate anemic blood conditions, lower hematocrit $(H t)$ and volume fraction of RBCs $\left(F_{R B C}\right)$, mentioned in Table 1, are factored into equation 4 and subsequently into equation 5 . The resulting values of $\mu_{\text {a.dermis }}$ are shown in Figure 2 (circles). Initial observation shows a difference in $\mu_{a}$ values in the green and red wavelenghts.

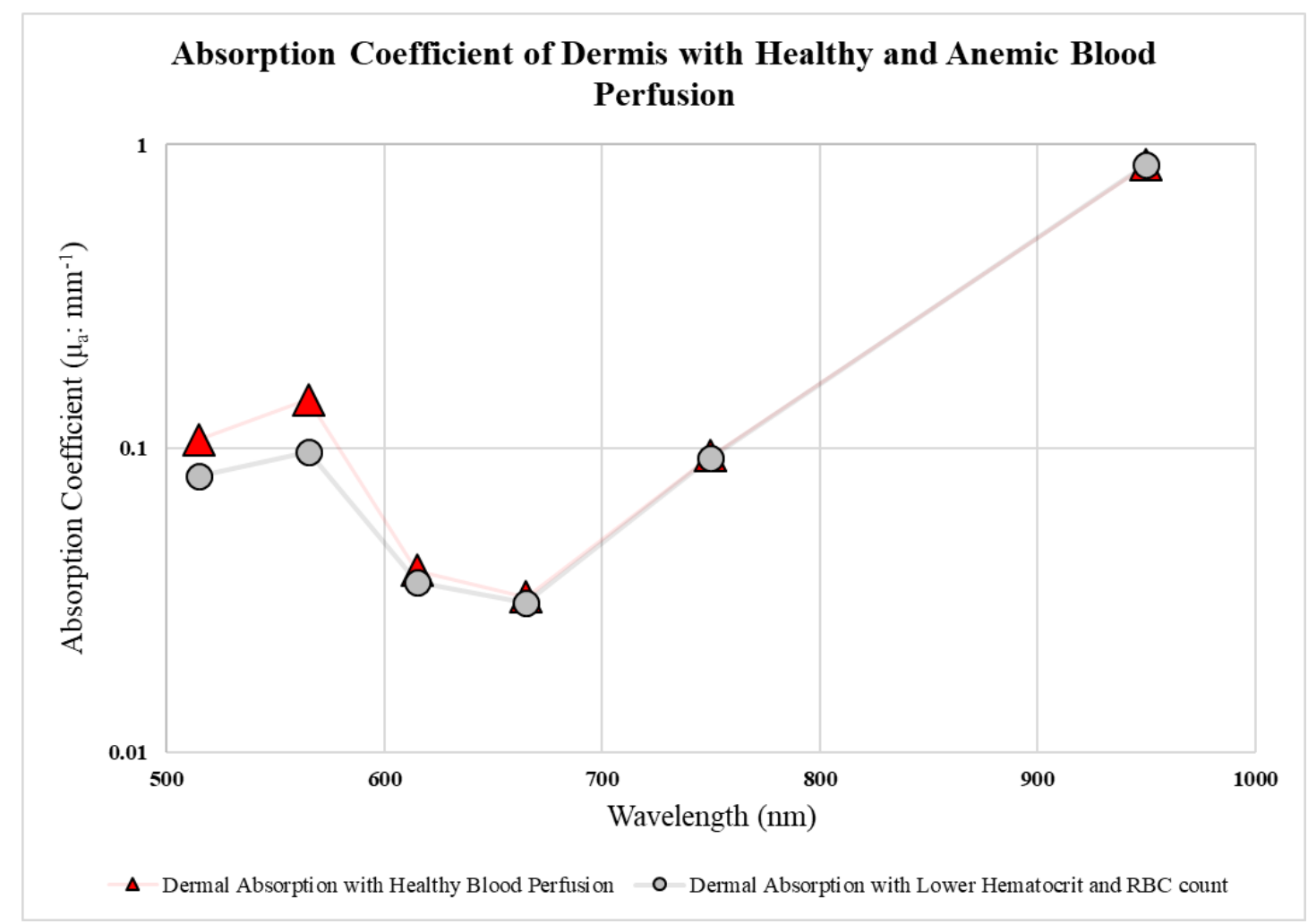

Figure 2. The lower values of hematocrit and volume fraction of RBCs are characteristic of anemic blood conditions. Once these contributions are considered, the absorption properties of the dermis layer $\left(\mu_{\text {a.dermis }}\right)$ perfused with healthy and anemic blood is calculated at the six wavelengths of interest. Higher absorption in the near-infrared region is attributed to water absorption.

\subsection{Scattering Coefficients of the Skin Layers}

Across multiple studies, there is a weak agreement for the reduced scattering coefficient values for the epidermis layer. The distribution of these values range between $10 \mathrm{~cm}^{-1}$ and $100 \mathrm{~cm}^{-1}$. Among the studies considered ${ }^{4,24-26}$ for ascertaining the reduced scattering coefficient of the epidermis $\left(\mu_{\text {s.epidermis }}^{\prime}\right)$, the optical properties are not reported for near-infrared wavelengths for the Svaasand et al. $(1995)^{26}$ and Jacques $(2013)^{4}$ studies. In the study by Salomatina et al. (2006), ${ }^{25}$ sample preparation (rinsing with PBS and rehydration with saline solution) for measurement of optical properties is speculated to cause the underestimation of the values. ${ }^{8}$ Therefore, the 
values reported by Altshuler et al. ${ }^{24}$ are reasoned to be the best fit for this current study. Contributing to this choice is also the fact that these values were extended from the widely accepted empirical relationship ${ }^{26}$ into the near-infrared region. The scattering coefficient $\left(\mu_{\text {s.epidermis }}\right)$ was calculated using the anisotropy, $g=0.82 .{ }^{27}$

The reduced scattering coefficient for the dermis layer was measured from excised samples previously. ${ }^{28}$ As the sample preparation did not involve washing and the measurement was done using accepted techniques, measurements using integrating spheres and inverse MC techniques. These measures are in agreement with Jacques' data, ${ }^{4}$ so a combination of both sets at visible and near-infrared wavelengths are used in our study for the reduced scattering coefficient value of the dermis layer $\left(\mu_{s . d e r m i s}^{\prime}\right)$. Anisotropy factor of $g=0.9^{27}$ is used to calculate the scattering coefficient, where necessary. The reduced scattering coefficient values of both layers, the epidermis and dermis, used in this study are shown in Figure 3 with the corresponding anisotropy factors.

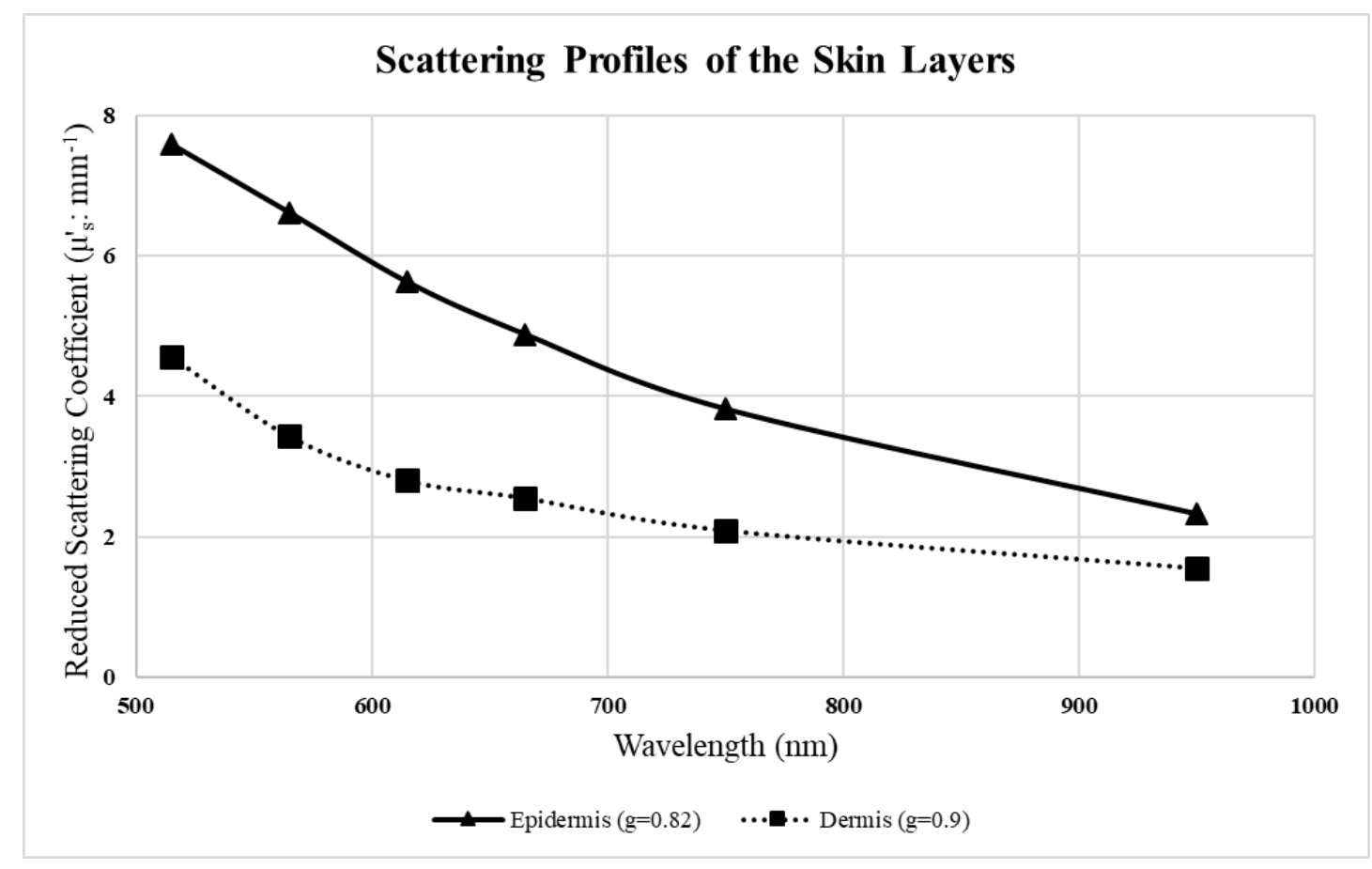

Figure 3. The epidermis and dermis layers' reduced scattering coefficients $\left(\mu_{s}^{\prime}\right)$ are adopted from literature ${ }^{22,24,28}$ after careful consideration. These values can be converted, if required, to scattering coefficients $\left(\mu_{s}\right)$ using the given anisotropy factors adopted from Karsten and Smit (2012). ${ }^{27}$

\subsection{Monte Carlo methods}

In order to execute the MC simulations of photon transport through the tissue layers, the refractive indices and thickness of the layers are required. The refractive indices of the two layers are set at $\eta_{\text {epidermis }}=1.34$ and $\eta_{\text {dermis }}=1.4 .{ }^{29}$ Thickness measurements $\left(d_{\text {epidermis }}=0.4 \mathrm{~mm}, d_{\text {dermis }}=3.48 \mathrm{~mm}\right)$ used in for the models are average measurements from in vivo ultrasound measurements we obtained from 17 participants for a previous study at the Great Western Hospital (Swindon, UK) using an 18-MHz probe. ${ }^{30}$ The thickness of the skin layers remains constant for all 72 simulation combinations of optical properties and wavelengths of interest.

The Virtual Photonics tool, Virtual Tissue Simulator, provides tools for photon-tissue interaction analysis. One such tool for MC simulations is known as Monte Carlo Command Line (MCCL). ${ }^{31}$ It allows for custom, structured input files to calculate and output recorded values from user-defined detectors. The input files, known as "infiles", are text files containing the input parameters and simulation settings, nature and position of the source, tissue layer definition (geometrical and optical properties) and detector definitions. In this study, the MC simulations are performed with $10^{6}$ photons and the following detectors (chosen from other available detectors) record the photon interactions. 
1. The total energy absorbed in the tissue model is measured with the detector, "ATotal."

2. The energy deposited within the tissue is recorded as a function of cylindrical coordinates (rho, $\rho$ ) and depth along the positive Z-axis $(z)$. The detector is referred to as "AOfRhoAndZ."

3. The detector that is of most significance to our current study is the reflected energy to the surface of the model. The "ROfRho" measuring reflected energy is defined as a function of cylindrical coordinates $(\rho)$.

4. Referred to as "ROfXAndY" in the MC workflow, the reflected photons from the tissue are captured as a function of Cartesian coordinates $(X, Y)$ on the surface of the model.

5. The detector "TOfRho" is similar to the ROfRho detector but placed at the bottom of the model to record transmission through the tissue.

6. Similar to the ROfXAndY detector, the "TOfXAndY" detector captures the energy transmitted through the model as a function of Cartesian coordinates $(X, Y)$.

7. Finally, to visualize the transport of photons in the tissue layers, the detector "FluenceOfRhoAndZ" is defined. This detector measures the fluence of energy in the tissue model as a function of cylindrical coordinates and depth $(\rho, z)$.

The sources are defined as directional point sources, emitting photons in the downward, positive $\mathrm{Z}$ direction $(+z)$. The input power is set at $5 \mathrm{~mW}$ which makes the photons skin safe at all wavelengths. A pseudorandom number generator, Marsenne Twister, is used in the simulations with a discretely defined absorption weighting method. The phase function applied is the Henyey-Greenstein function and the simulation accounts for Fresnel interactions at all boundaries. All these factors are defined at the beginning of the infiles to set up the simulation. The tissue geometry and optical properties are subsequently set and the simulations are executed. The 72 combinations of optical properties at multiple wavelengths, combined with the above detectors for each scenario result in a total of 432 image results. The input files used for MC assessment, along with the respective results are accessible on the Cranfield Online Research Data (CORD) repository. ${ }^{32}$

\section{RESULTS AND DISCUSSION}

The primary objective of this research was to use MC methods of assessing photon transport for detecting anemic blood conditions in the skin. The optical properties of the epidermis and dermis layers were set to replicate variable melanin concentration in the skin layers and blood conditions. The assessment was made at six wavelengths across the visible and near-infrared regions in order to identify keys for differentiating healthy and anemic blood perfusion in the dermis layer. As stated previously, these combinations and the detectors used in the simulations resulted in 432 images that are available on the CORD repository ${ }^{\ddagger} .32$

The simulations results were recorded and saved as individual files in a pre-defined folder. The results can be visualized using the MCCL tool's MATLAB code (provided with the download package). Each simulation generated six image outputs. Of the 72 simulation scenarios, the results of type II skin type and anemic blood perfusion assessed at $665 \mathrm{~nm}$ are shown in Figure 4:

- Absorbed/deposited energy (Fig. 4 (A)) and fluence (Fig. 4 (B)) in the tissue volume;

- Surface distribution of photons transmitted (Fig. 4 (C)) and reflected (Fig. 4 (D)) from the tissue;

- Transmission through the tissue (Fig. 4 (E)) as a function of cylindrical coordinates $(\rho)$;

- Reflected energy from the tissue (Fig. $4(\mathrm{~F})$ ) as a function of cylindrical coordinates $(\rho)$

The reflected photons propagate through the epidermis twice between the source and detector. Although highly forward scattering, the epidermis layer also absorbs photons with an increasing proportionality to the concentration of melanin in the layer (Fig. 4 (A), Fig. 5). The fluence, calculated with regards to the smallest unit of surface area in the volume of the tissue and integrated over time, shows the transport of the photons (Figure $4(\mathrm{~B})$ ). The photons travel dominantly forward, scattered away from the center $(\rho=0)$ and deposit energy through the volume of the tissue. Both the epidermis and dermis layers are highly forward scattering, and in the cases of increasing wavelength or reducing melanin concentration, the photons travel further into the tissue. Although the surface distribution of transmitted and reflected photons (Figure 4 (C, D)) does not reveal

\footnotetext{
${ }^{\ddagger}$ Accessible at https://doi.org/10.17862/cranfield.rd.11317187
} 

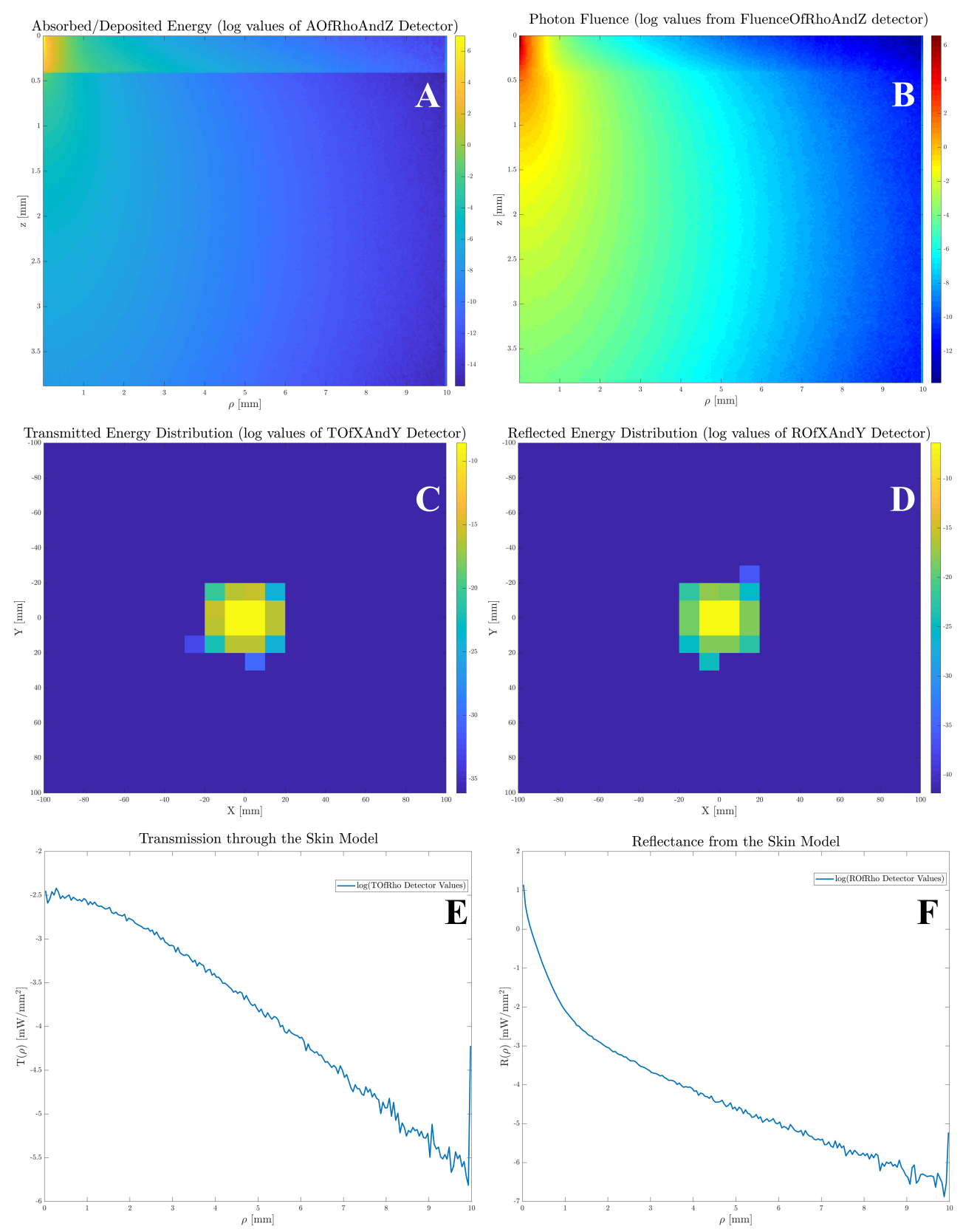

Figure 4 . The results of simulating $10^{6}$ photons through skin type II $\left(f_{\text {melanin }}=0.045\right)$ and anemic blood conditions in the dermis layer at $665 \mathrm{~nm}$ wavelength are presented. Energy deposited in the volume of the tissue (A), fluence of photons in the tissue (B), transmitted (C) and reflected (D) photons at the tissue-air interfaces show the interaction of photons with the tissue and best sites for detection of energy. The transmitted and reflected energy show the energy received on the surface detectors as a function of cylindrical coordinates, $\rho$.

any clear insights for source-detector separation and placements, we observe that a large detector separation is not necessary in the case of assessing photon transport through tissues.

Of the detectors and results output from the MC simulations, the results further examined for understanding photon transport in anemic blood perfused skin are the transmitted and detected photons recorded as a function of cylindrical coordinates $(\rho)$. As expected, the transmission (Figure $4(\mathrm{E})$ ) and reflection (Figure $4(\mathrm{~F})$ ) from 

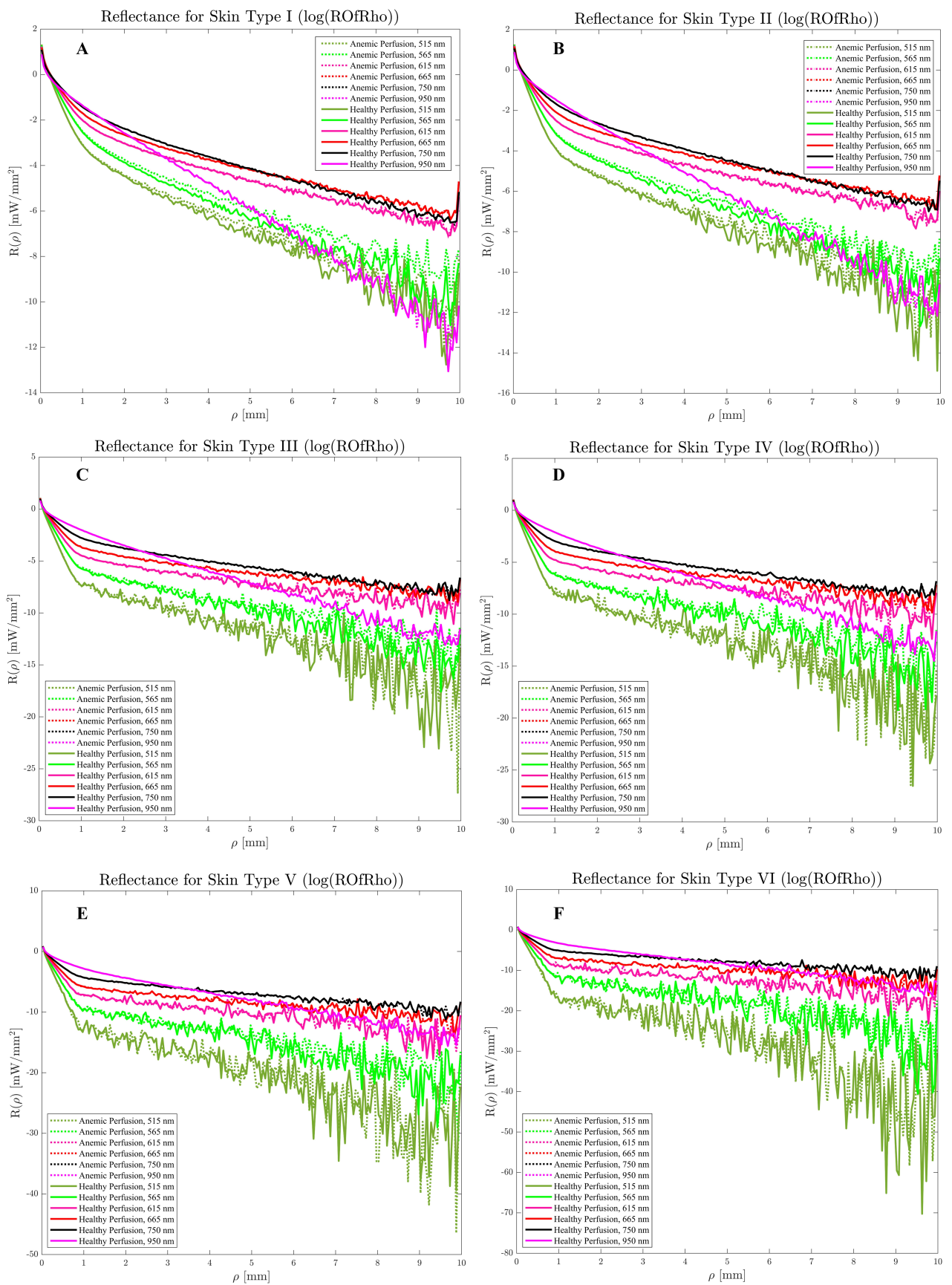

Figure 5. Reflectance from the tissue model is measured on the surface of the model by recording the photons and photon weights as a function of cylindrical coordinates $(\rho)$. Each plot (A-F) shows the $\log$ (ROfRho) for a particular skin type (constant $f_{\text {melanin }}$ ), showing healthy and anemic blood conditions at all 6 wavelengths.

the tissue model reduces with increasing distance from the point of incidence of the photons, $(\rho=0)$. The transmission results showed an expected trend of greater transmission in for lower melanin concentration and anemic conditions as the distribution of absorbing chromophores is lower in comparison to healthy blood conditions (As seen when combining blood characteristics (Table 1) with Eq. 4, 5). However, the difference when comparing healthy and anemic tissue transmission at a single wavelength and for the same skin type is a very 
small fraction that is not sufficient enough to provide a basis for designing devices. This difference reduces with increasing wavelength as the ability of photons of longer wavelengths to travel further into the tissue is higher. ${ }^{7}$ Realistically, the number of sites in the body for the measurement of transmitted energy.

We shift our focus to the reflectance measured in the simulations, as the primary objective of this research is to evaluate the reflectance-based detection of anemic blood conditions. In Figure 5, the reflectance from anemic tissue conditions are shown as a dotted line (...... ) and healthy tissue conditions are shown as a solid line (一). The analysis focuses on different blood conditions at different wavelengths but assessed specific to a particular skin type. Understanding the influence of melanin in the epidermis layer will be key to gaining information from the layers below. In this case, it is the dermis layer perfused with blood. Constant input power allows us to analyze the distribution of photons and the variation of reflected energy with increasing distance from the point of incidence (increasing $\rho$ ). An observation from all the six plots (Figure $5(\mathrm{~A}-\mathrm{F})$ ) is the distinctly different trend at $950 \mathrm{~nm}$ in comparison to the other wavelengths. The least reflectance is seen in the green wavelengths $(515$ $\mathrm{nm}, 565 \mathrm{~nm}$ ). The photons at these wavelengths are absorbed in the skin layers. ${ }^{7,33,34}$ The overall reflectance is least in the case of skin type VI (Fig. $5(\mathrm{~F})$ ), as the epidermis layer with the highest melanin concentration and distribution absorbs to the greatest degree, across all wavelengths (least values of $\log (\mathrm{ROfRho})$ ).

Conclusions regarding the variable reflectance of the various wavelengths for the different skin types can be made from this comparison. Across all skin types, the crossover between the other wavelengths and $950 \mathrm{~nm}$ reflectance is a key identifier for detecting blood contribution to the attenuation of the photons. This variable interaction has been previously and now widely used for oximeter measurements. ${ }^{34}$ The crossover also shifts farther from the point of incidence of the photons as the absorption by the skin layers increases. For instance, the crossover of the plots is approximately $2 \mathrm{~mm}$ away from the point of incidence for skin type I $(\rho=2)$ while it is beyond $4 \mathrm{~mm}$ for skin type VI $(\rho=4)$. This shift shows the increasing influence of the absorption and scattering of the photons due to melanin in the epidermis layer.

\section{CONCLUSION AND FUTURE WORK}

The research objective was to evaluate the possibility of detecting anemic conditions purely from perfused blood in the skin. The contributions of melanin in the epidermis and blood (hemoglobin being the primary chromophore) in the dermis layer. The optical properties for these layers considered these contributions and MC methods were used to analyze the two-layer models. The results showed the photon interactions and transport through the models, and the primary analysis looked at reflected energy from the skin. Our key inference is that, within the scope of this study, the two layers of the skin and associated optical properties do not provide conclusive information for detecting anemia. However, the influence of melanin in the epidermis layers and the contribution of blood conditions to the attenuation of the photons provide evidence of the variations in reflected and transmitted energy.

Not much separates healthy and anemic blood conditions when considering the skin layers alone. From the results of this study, we conclude that a full model including other tissues and blood vessels (arteries and veins) is the next step. In the case of the pulse oximeters, our initial comparison, the variable transmission and absorption of red and near-infrared wavelengths through an entire finger's width gives the oxygen saturation and heart rate. The presence of a blood vessel relatively close to the surface of the skin could provide sufficient variability to detect anemia, as light at red wavelengths is capable of interacting with the blood inside the vessels. ${ }^{13}$ Studies using image capture and processing ${ }^{35}$ are in progress but a combination of sensors and imaging algorithms will be a significant step forward to integrate this detection into point-of-care devices.

\section{ACKNOWLEDGMENTS}

This work was made possible through open-source software resources offered by the Virtual Photonics Technology Initiative, at the Beckman Laser Institute, University of California, Irvine. A special thank you to Dr Carole K Hayakawa (UC Irvine) for her assistance with Virtual Tissue Simulator (VTS) and Monte Carlo Command Line (MCCL). The authors are grateful to the IEEE Photonics Chapter for their travel grant that has made the presentation of this paper at SPIE Photonics West 2020 possible. A special mention to my doctoral colleagues who put me in touch with wonderful medical personnel who provided valuable clarifications. 


\section{REFERENCES}

[1] Billett, H. H., "Hemoglobin and Hematocrit," in [Clinical Methods: The History, Physical, and Laboratory Examinations], Walker, H. K., Hall, W. D., and Hurst, J. W., eds., ch. 151, 718-719, Butterworths, 3 ed. (1990).

[2] Fitzpatrick, T. B., "The validity and practicality of sun-reactive skin types I through VI," Archives of Dermatology 124(6), 869-871 (1988).

[3] Boudoux, C., "Introduction," in [Fundamentals of Biomedical Optics], ch. 1, 1-3, Blurb Incorporated (2016).

[4] Jacques, S. L., "Optical properties of biological tissues: A Review," Physics in Medicine and Biology 58(11), R37-R61 (2013).

[5] Boudoux, C., "Light Transport in Tissue," in [Fundamentals of Biomedical Optics], ch. 11, 237-260, Blurb Incorporated (2016).

[6] Henyey, L. G. and Greenstein, J. L., "Diffuse radiation in the Galaxy," The Astrophysical Journal 93, 70-83 (1941).

[7] Anderson, R. R. and Parrish, J. A., "The Optics of Human Skin," The Journal of Investigative Dermatology $\mathbf{7 7}(1), 13-19$ (1981).

[8] Mignon, C., Tobin, D. J., Zeitouny, M., and Uzunbajakava, N. E., "Shedding light on the variability of optical skin properties: finding a path towards more accurate prediction of light propagation in human cutaneous compartments," Biomedical Optics Express 9(2), 852-872 (2018).

[9] Chandrasekhar, S., [Radiative transfer], Dover Publications, Inc. (New York, NY) (1960).

[10] Wang, L. V. and Wu, H., "Monte Carlo Modeling of Photon Transport in Biological Tissue," in [Biomedical Optics: Principles and Imaging], ch. 3, John Wiley \& Sons, Inc., Hoboken, New Jersey (2007).

[11] Wang, L., Jacques, S. L., and Zheng, L., "MCML - Monte Carlo modeling of light transport in multilayered tissues," Computer Methods and Programs in Biomedicine 47(2), 131-146 (1995).

[12] Prahl, S. A., Keijzer, M., Jacques, S. L., and Welch, A. J., "A Monte Carlo model of light propagation in tissue," Dosimetry of Laser Radiation in Medicine and Biology 5, 102-111 (1989).

[13] Nilsson, H. and Nilsson, G. E., "Monte Carlo simulations of light interaction with blood vessels in human skin in the red-wavelength region," in [Proceedings in SPIE 3252, Optical Diagnostics of Biological Fluids III], Priezzhev, A. V., Asakura, T., and Briers, J. D., eds., 3252, 44-53, SPIE (1998).

[14] Meglinskii, I. V., "Monte Carlo simulation of reflection spectra of random multilayer media strongly scattering and absorbing light," Quantum Electronics 31(12), 1101-1107 (2001).

[15] Meglinski, I. V. and Matcher, S. J., "Quantitative assessment of skin layers absorption and skin reflectance spectra simulation in the visible and near-infrared spectral regions," Physiological Measurement 23, 741-753 (2002).

[16] Patwardhan, S. V., Dhawan, A. P., and Relue, P. A., "Monte Carlo simulation of light-tissue interaction: Three-dimensional simulation for trans-illumination-based imaging of skin lesions," IEEE Transactions on Biomedical Engineering 52(7), 1227-1236 (2005).

[17] Palmer, G. M., Zhu, C., Breslin, T. M., Xu, F., Gilchrist, K. W., and Ramanujam, N., "Monte Carlo-based inverse model for calculating tissue optical properties. Part II: Application to breast cancer diagnosis.," Applied Optics 45(5), 1072-1078 (2006).

[18] Palmer, G. M., Zhu, C., Breslin, T. M., Xu, F., Gilchrist, K. W., and Ramanujam, N., "Monte Carlo-based inverse model for calculating tissue optical properties. Part II: Application to breast cancer diagnosis.," Applied Optics 45(5), 1072-1078 (2006).

[19] Maeda, T., Arakawa, N., Takahashi, M., and Aizu, Y., "Monte Carlo Simulation of Spectral Reflectance Using a Multilayered Skin Tissue Model," Optical Review 17(3), 223-229 (2010).

[20] Chen, S., Yi, J., Liu, W., Backman, V., and Zhang, H. F., "Monte Carlo Investigation of Optical Coherence Tomography Retinal Oximetry," IEEE Transactions on Biomedical Engineering 62(9), 2308-2315 (2015).

[21] Zhu, C. and Liu, Q., "Review of Monte Carlo modeling of light transport in tissues," Journal of Biomedical Optics 18(5), 50902 1-13 (2013).

[22] Jacques, S. L. and McAuliffe, D. J., "The melanosome: Threshold temperature for explosive vaporization and internal absorption coefficient during pulsed laser irradiation," Photochemistry and Photobiology 53(6), 769-775 (1991). 
[23] Saidi, I. S., Transcutaneous optical measurement of hyperbilirubinemia in neonates, PhD thesis, Rice University (1992).

[24] Altshuler, G., Smirnov, M., and Yaroslavsky, I. V., "Lattice of optical islets: A novel treatment modality in photomedicine," Journal of Physics D: Applied Physics 38, 2732-2747 (2005).

[25] Salomatina, E., Jiang, B., Novak, J., and Yaroslavsky, A. N., "Optical properties of normal and cancerous human skin in the visible and near-infrared spectral range," Journal of Biomedical Optics 11(6), 064026 1-9 (2006).

[26] Svaasand, L. O., Norvang, L. T., Fiskerstrand, E. J., Stopps, E. K. S., Berns, M. W., and Nelson, J. S., "Tissue parameters determining the visual appearance of normal skin and port-wine stains," Lasers in Medical Science 10, 55-65 (1995).

[27] Karsten, A. E. and Smit, J. E., "Modeling and verification of melanin concentration on human skin type," Photochemistry and Photobiology 88, 469-474 (2012).

[28] Simpson, R. C., Kohl, M., Essenpreis, M., and Cope, M., "Near-infrared optical properties of ex vivo human skin and subcutaneous tissues measured using the Monte Carlo inversion technique," Physics in Medicine and Biology 43, 2465-2478 (1998).

[29] Tuchin, V. V., "Methods and Algorithms for the Measurement of the Optical Parameters of Tissues," in [Tissue Optics: Light Scattering Methods and Instruments for Medical Diagnosis], Tuchin, V. V., ed., ch. 2, 143-256, SPIE (The Society of Photo-Optical Instrumentation Engineers), Bellingham, Washington, 2 ed. (2007).

[30] Kallepalli, A., McCall, B., James, D. B., Junaid, S., Halls, J., and Richardson, M. A., "Optical investigation of three-dimensional human skin equivalents: A pilot study," Journal of Biophotonics 13, e20190053 (2020).

[31] Virtual Photonics, "Monte Carlo Command Line (MCCL) Getting Started," (2019). Access URL: https://github.com/VirtualPhotonics/VTS/wiki/MCCL-Getting-Started, Last Accessed On: 31-12-2019.

[32] Kallepalli, A. and James, D. B., "Monte Carlo simulation results for anaemia detection: Repository on Cranfield Online Research Data (CORD)," (2019). [doi:10.17862/cranfield.rd.11317187].

[33] Severinghaus, J. W. and Koh, S. O., "Effect of anemia on pulse oximeter accuracy at low saturation," Journal of Clinical Monitoring 6(2), 85-88 (1990).

[34] Jubran, A., "Pulse oximetry," Intensive Care Medicine 30(11), 2017-2020 (2004).

[35] Mannino, R. G., Myers, D. R., Tyburski, E. A., Caruso, C., Boudreaux, J., Leong, T., Clifford, G. D., and Lam, W. A., "Smartphone app for non-invasive detection of anemia using only patient-sourced photos," Nature Communications 9, 1-10 (dec 2018). 